\section{Potential routes of spread of Zika virus to the Middle East, North Africa and Asia: action must be taken}

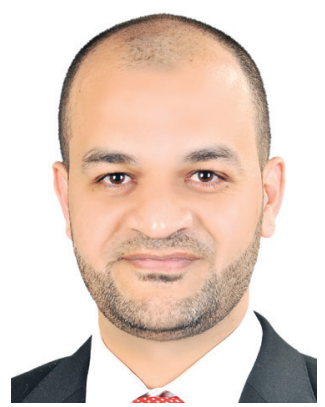

\author{
“Zika virus and its infection are \\ among the most recent \\ significant viral security threats \\ for global public health.”
}

Hatem Zayed ${ }^{*}, 1$, George Priya Doss C ${ }^{2} \&$ Mohamed Ezzat El Zowalaty**,3

First draft submitted: 12 December 2016; Accepted for publication: 25 January 2017; Published online: 5 April 2017

Zika virus (ZIKV) and its infection are among the most recent significant viral security threats for global public health. ZIKV causes severe international concern due to its pandemic potential, economic burden, morbidity and mortality. ZIKV is a neurotropic, sexually transmitted Flavivirus and is a member of the Flaviviridae family, related to yellow fever, dengue (DEN), West Nile and Spondweni viruses. It is an enveloped virus with a single-strand positive sense RNA consisting of a 10,794-nucleotide genome. As a mosquito-borne virus, ZIKV is transmitted by many Aedes spp. mosquitoes, including Aedes africanus, Aedes luteocephalus, Aedes hensilli, Aedes albopictus and Aedes aegypti [1]. ZIKV disease is a zoonotic arboviral nationally notifiable infection where human and nonhuman primates are the main reservoirs, and anthroponotic (human-to-vector-to-human) transmission may occur during outbreaks [2]. The viral transmission occurs through transfusion of infected blood, sexual intercourse and from infected mothers to their fetus during pregnancy. ZIKV is generally eliminated from the body after a few weeks although viral RNA may persist for longer periods in the semen after infection suggesting a high risk for sexual transmission [3]. Upon infection, ZIKV has been associated with congenital microcephaly, neurological complications including Guillain-Barré syndrome, meningoencephalitis and acute myelitis $[4,5]$ and autoimmune complications [6]. Approximately 134 cases in Brazil have been confirmed to have microcephaly, and the remaining 2165 cases are under investigation [7].

ZIKV was first identified in rhesus macaque during sylvatic yellow fever surveillance in the Zika Forest, Uganda, in 1947 and was first reported in humans in 1952 [8]. Before 2006, only 14 cases of human ZIKV disease were reported,

\section{KEYWORDS}

- Aedes $\bullet$ arboviruses $\bullet$ MENA region - mosquito • surveillance $\bullet$ vectorborne $\bullet$ Zika virus $\bullet$ zoonotic 


\section{"The increased connectedness of populations across the globe creates favorable conditions for the rapid transmission of new emerging diseases such as Zika virus disease."}

including one case which was a laboratoryacquired infection [9-13], and from 2007 to 2016, the disease has been reported in more than 40 countries. At the end of 2016, the number of Zika cases is escalating with an incidence rate of $71.23 \%$ and with a total of 534,620 suspected cases, 176,239 confirmed cases, 5233 imported cases, 18 deaths and 2443 confirmed congenital syndromic cases in the Americas [14]. This scenario triggered the US CDC and the NIH to declare that the ZIKV is at a 'pandemic' level, and the CDC issued a warning to pregnant women to avoid traveling to ZIKV-affected regions [2].

The Aedes species is widely distributed throughout the world, including Arab region countries and the Middle East. Among the routes available to the virus to cause a global pandemic and spread quickly to Asia, Africa and Europe, it is through the annual gathering in Mecca where more than two million of Muslims gather from across the globe. Here, we discuss the potential routes which could be responsible for the global Zika pandemic, and strategies of governmental health officials, the WHO and the public could adopt to limit spread of the virus.

Potential routes for global ZIKV pandemic

The increased connectedness of populations across the globe creates favorable conditions for the rapid transmission of new emerging diseases such as ZIKV disease. The Kingdom of Saudi Arabia issues millions of visas for Muslims coming from all over the world for practicing Umrah (pilgrimage to Holy Mecca, which may be performed by Muslims at any time of the year). The Hajj (annual pilgrimage, which is performed by Muslims only once a year at specific time) is an ongoing annual gathering event. In this annual spiritual gathering, more than a million of pilgrims visit the holy locations in Mecca, Saudi Arabia. This gathering includes Muslims from across the globe, including South Americans, Europeans and Asians. In addition to this, a significant number of the expatriate workers from South America, Africa and Asia who reside in Saudi Arabia, Qatar, Bahrain, Kuwait, Oman and United Arab Emirates annually visit their home countries. A silent reservoir of ZIKV in Arab countries, Asia and Africa could be immunologically naive individuals who do not have adequate knowledge about the mode of transmission or who are not able to protect themselves from ZIKV. There are a few factors that are favorable to help spread ZIKV during Umrah and Hajj seasons, including elevated climatic temperatures, which may exceed $100^{\circ} \mathrm{F}$, and sexual transmission. Earlier and recent reports of ZIKV through male sex partner semen from Florence (Italy) and Dallas (Texas, USA) made the CDC declare ZIKV disease as sexually transmitted disease $[15,16]$. The CDC has advised travelers from infected countries to use condoms or to avoid sexual contact with the infected males [17]. These gathering events could be the nuclei for infectious and communicable disease transmission. Therefore, special attention must be given to individuals taking part in these events to limit the spread of ZIKV disease and avoid the looming risk of a global pandemic.

There are plenty of potential niches in the Middle East and North African countries where the Aedes species mosquito could find fertile environments to breed, such as in stagnant water of uncovered containers, toilet tanks, flower vases, buckets and discarded bags. In addition, wet areas around air-conditioning systems, which are commonly used in Saudi Arabia and other Gulf countries and in Asia due to the hot climate, may be potential breeding areas. Mosquitoes gather around these environments representing high risk for transmission of ZIKV as well as DEN viruses.

Therefore, raising awareness about the spread of the disease should be a priority, and warnings need to be officially issued to the areas where the virus has reached the pandemic level. These areas should also be cautiously monitored for all incoming travelers, whether they are domestic or international travelers. Several methods could be used to provide protection to the pilgrims in the Hajj season, including installing ovitraps to monitor insects that are known to transmit ZIKV, the use of barriers like screens, closed doors and windows, the use of mosquito nets and spraying or fogging with insecticides in areas that are suspected of the presence of the Aedes mosquito.

There are little data available on the range of zoonoses circulating in the Middle East and North African countries. This includes a lack of knowledge on the presence of medically important arthropod vectors, while endemicity for a range of zoonoses is based on knowledge from other countries in the Arabian Peninsula with similar climatic and socioeconomic conditions. 
More importantly, the increasing demand for expatriates and livestock is expected to increase in the coming years due to increasing labor requirements in urbanization, among others related to the infrastructure needed for the World Championships Soccer in Qatar 2022. These increasing requirements will continue with escalating risks for the introduction of arboviruses including ZIKV vectors into the region. In addition, the championships will attract visitors from different global regions including ZIKV-affected areas such as Central and South America, the Caribbean, Asia and Africa, imposing an additional demand on the public health systems.

Climate changes create favorable conditions for the establishment of disease vectors, such as Aedes spp., in new geographic regions supporting local transmission of ZIKV. The perturbation between ecological balances, including global climate changes and increased international travel, helps spread of ZIKV. The majority of emerging infectious diseases are zoonotic such as ZIKV infection with the involvement of wildlife, which may have serious effects on human health. Humans, animals and the environment are interconnected and will have multidirectional impact on each other. Surveillance for viral pathogens and vector-borne infections in animal species and wildlife are imperative intervention measures that will help reduce and control the spread of emerging arboviruses including ZIKV.

\section{Conclusion \& future perspective}

Although ZIKV is known to circulate in Africa, the Americas and Asia, there is uncertainty as to what the pandemic level it is expected to reach and what the actual risks of the virus are to endanger human health in the absence of licensed vaccines and specific effective antiviral agents. Therefore, it is of great importance to conduct regular surveillance on ZIKV disease among domestic travelers coming or known to be in contact with people from epidemic and infected areas. This can be quickly performed using sensitive molecular diagnostic techniques such as conventional or real-time reverse transcription PCR to investigate early infection and detect viral RNA in body fluids such as urine, cerebrospinal fluid, semen or serum [2]. Concerted global and national efforts to tackle the disease should be advocated in ZIKV-affected regions as well as in other high-risk countries. This is important since one in five people infected with ZIKV become symptomatic, and most cases (about $80 \%)$ are asymptomatic with a self-limiting febrile illness [18]. Serological techniques detecting virus-specific anti-ZIKV IgM provide timely prenatal intervention to infected pregnant women. These approaches will ensure that the virus is contained and will avoid further spread of the virus. However, these efforts cannot be achieved without concerted efforts from individual cooperations among the governmental health authorities and international organizations, such as the WHO, to work hand in hand in order to avoid the spread of this disease and save lives now and in the future. To date, there are no clinically approved vaccines available for the prophylaxis against ZIKV infection as well as many other flaviviruses. In addition, there is no specific chemotherapeutic antiviral agent currently available for treatment. There are two DNA vaccines against ZIKV that are under development in clinical trial phases [19], although there are no DNA vaccines yet available for clinical use in humans against any infectious disease. Therefore, further epidemiological and control studies are critical to implement the most appropriate prevention measures which are crucial to reduce the incidence of infection and will reduce transmission. The use of insecticides and mosquito repellent is important but should be regulated to avoid spread of resistance. The recent use and application of biological control measures such as Wolbachia in reducing transmission of dengue virus could pave the way for use in ZIKV transmission reduction as well [20-23]. More research studies are required to investigate the use and benefits of biological control measures and search for additional biological control agents against ZIKV to combat arboviral spread in parallel to $\mathrm{ZIKV}$ vaccine initiatives.

\section{Financial \& competing interests disclosure}

The authors have no relevant affiliations or financial involvement with any organization or entity with a financial interest in or financial conflict with the subject matter or materials discussed in the manuscript. This includes employment, consultancies, honoraria, stock ownership or options, expert testimony, grants or patents received or pending, or royalties.

No writing assistance was utilized in the production of this manuscript.

\author{
"Climate changes create \\ favorable conditions for \\ the establishment of \\ disease vectors, such as \\ Aedes spp., in new \\ geographic regions \\ supporting local \\ transmission of Zika virus."
}




\section{References}

1 Campos GS, Bandeira AC, Sardi SI. Zika virus outbreak, Bahia, Brazil. Emerg. Infect. Dis. 21(10), 1885-1886 (2015).

2 Centers for Disease Control and Prevention. Zika virus clinical evaluation and disease. www.cdc.gov/zika/hc-providers

3 Atkinson B, Thorburn F, Petridou C et al. Presence and Persistence of Zika Virus RNA in Semen, United Kingdom, 2016. Emerg. Infect. Dis. 23(4), doi:10.3201/ eid2304.161692 (2017)

4 Anaya JM, Ramirez-Santana C, SalgadoCastaneda I et al. Zika virus and neurologic autoimmunity: the putative role of gangliosides. BMC Med. 14, 49 (2016).

5 Mecharles S, Herrmann C, Poullain P et al. Acute myelitis due to Zika virus infection. Lancet 387(10026), 1481 (2016).

6 Carteaux G, Maquart M, Bedet A et al. Zika virus associated with meningoencephalitis. N. Engl. J. Med. 374(16), 1595-1596 (2016).

7 Triunfol M. A new mosquito-borne threat to pregnant women in Brazil. Lancet Infect. Dis. 16(2), 156-157 (2016).

8 Dick GW, Kitchen SF, Haddow AJ. Zika virus. I. Isolations and serological specificity. Trans. R. Soc. Trop. Med. Hyg. 46, 509-520 (1952).

9 Simpson DI. Zika virus infection in man Trans. R. Soc. Trop. Med. Hyg. 58, 335-338 (1964).
10 Moore DL, Causey OR, Carey DE et al. Arthropod-borne viral infections of man in Nigeria, 1964-1970. Ann. Trop. Med. Parasitol. 69, 49-64 (1975).

11 Fagbami AH. Zika virus infections in Nigeria: virological and seroepidemiological investigations in Oyo State. J. Hyg. (Lond.) 83, 213-219 (1979).

12 Filipe AR, Martins CM, Rocha $\mathrm{H}$. Laboratory infection with Zika virus after vaccination against yellow fever. Arch. Gesamte Virusforsch. 43, 315-319 (1973).

13 Olson JG, Ksiazek TG, Suhandiman T. Zika virus, a cause of fever in Central Java, Indonesia. Trans. R. Soc. Trop. Med. Hyg. 75 , 389-393 (1981).

14 Pan American Health Organization/World Health Organization. Zika Suspected and Confirmed Cases Reported by Countries and Territories in the Americas Cumulative cases, 2015-2016 (Updated as of 22 December 2016). PAHO/WHO, Washington, DC, USA (2016). www.paho.org/hq/index.php?option

15 Sikka V, Chattu VK, Popli RK et al. The emergence of zika virus as a global health security threat: a review and a consensus statement of the INDUSEM Joint working Group (JWG). J. Glob. Infect. Dis. 8(1), 3-15 (2016).

16 Dallas County Health and Human Services. DCHHS reports first Zika virus case in Dallas County acquired through sexual transmission. www.dallascounty.org/department/hhs
17 Oster AM, Brooks JT, Stryker JE et al. Interim Guidelines for Prevention of Sexual Transmission of Zika Virus, United States, 2016. MMWR Morb. Mortal Wkly Rep. 65(5), 120-121 (2016).

18 Pastula DM, Smith DE, Beckham JD, Tyler KL. Four emerging arboviral diseases in North America: Jamestown Canyon, Powassan, chikungunya, and Zika virus diseases. J. Neurovirol. 22(3), 257-260 (2016).

19 Morrison C. DNA vaccines against Zika virus speed into clinical trials. Nat. Rev. Drug Discov. 15(8), 521-522 (2016).

20 Callaway E. Rio fights Zika with biggest release yet of bacteria-infected mosquitoes. Nature 539(7627), 17-18 (2016).

21 Caragata EP, Dutra HL, O’Neill SL, Moreira LA. Zika control through the bacterium Wolbachia pipientis. Future Microbiol. 11, 1499-1502 (2016).

22 Aliota MT, Peinado SA, Velez ID, Osorio JE. The wMel strain of Wolbachia reduces transmission of Zika virus by Aedes aegypti. Sci. Rep. 6, 28792 (2016)

23 Dutra HL, Rocha MN, Dias FB et al. Wolbachia blocks currently circulating Zika virus isolates in Brazilian Aedes aegypti mosquitoes. Cell Host Microbe 19(6), 771-774 (2016). 